\title{
The Constant-Volume Heat Capacity of
}

\section{Gaseous Tetrafluoromethane}

\author{
YU-TANG HWANG and JOSEPH J. MARTIN \\ The University of Michigan, Ann Arbor, Michigan
}

Up to the present time there does not appear to have been any calorimetric measurements of the heat capacity of gaseous tetrafluoromethane. Consequently this compound has been included in a continuing program of study of the constant-volume heat capacity $C_{V}$ of gases at the University of Michigan.

The equipment for making these measurements consists primarily of an 8-in. diameter thin-wall stainless-steel sphere mounted in an evacuated chamber with a heat shield surrounding the sphere. The sphere is equipped with a platinum heating coil, which doubles as a resistance thermometer, and a small motor-driven fan that circulates the charge of gas inside the sphere. Complete details are described elsewhere $(4,7,9)$, and only the calibration procedure with tetrafluoromethane is covered here.

\section{CALIBRATION OF THE CALORIMETER}

When heat is added to a charge of gas in a container of fixed volume, it is necessary to know the heat capacity of the container and its internal fittings so that proper assignment may be made of that fraction of the heat going into the gas. Also, since it is extremely difficult to attain perfectly adiabatic conditions, some estimate must be made of the heat transfer to the surroundings. The latter is determined by measuring drift rates of the temperature within the container before and after heating. The former may be determined in two ways: by taking some measurements on a gas of known heat capacity, so that the heat capacity of the calorimeter may be obtained by subtracting the known gas heat capacity from the gross heat capacity, and by an extrapolation technique using a gas whose constant-volume heat capacity varies little with density.

The second technique was used in this case. Since tetrafluoromethane has a low critical temperature of $227.5^{\circ} \mathrm{K}$., and since the data were to be taken above $300^{\circ} \mathrm{K}$, all runs were made at reduced temperatures greater than 1.3 . The variation of $C_{V}$ with density is a function of the curvature of the isometrics of the PVT data, namely $d^{2} P / d T^{2}$. At reduced temperatures above 1.3 this second derivative becomes very small, as the isometrics are practically straight. Thus $C_{V}$ at any density is practically equal to $C V$ which is a function of temperature only (the refers to zero density). One may then write

Yu-Tang Hwang is with Pure Oil Company, Crystal Lake, Illinois.

$$
C_{\text {calr }}=C_{\mathrm{gr}}-m C_{V} \text { * }
$$

where calr refers to the calorimeter, gr refers to the gross heat capacity, and $m$ is the mass of gas charged with $C_{V}$ on a unit mass basis. Suppose $C_{V}$ is not yet known, but some approximation to it such as $C_{V}{ }^{*}$ app can be made. $W$ ith a correction term one may write

$$
C_{V}^{*}=C_{V}^{*}{ }_{\text {app }}+C_{V}{ }^{*} \text { corr }
$$

Combining (1) and (2) and rearranging one obtains

$$
C_{\mathrm{gr}}-m C_{V}{ }^{*}{ }_{\text {app }}=C_{\text {calr }}+m C_{V}{ }^{*}{ }_{\text {corr }}
$$

If now $C_{\mathrm{gr}}$ is measured at different densities (that is different $m$ 's) at a given temperature, where $C_{V}{ }^{*}$ app, $C_{\text {calr }}$, and $C_{V}$ corr are all fixed, it follows that $C_{\mathrm{gr}}-m C_{V}{ }^{*}$ app is linear in $m$. Plotting $C_{g r}-m C_{V}{ }^{*}$ app vs. $m$ one gets a linear extrapolation to zero density at which point $C_{\text {calr }}$ becomes identical to $C_{\mathrm{gr}}$, which is the desired heat capacity of the calorimeter.

Figure 1 is just such a plot for tetrafluoromethane where $C_{V}{ }^{*}$ app has been taken to be $C_{V}{ }^{*}$ (statistical), a heat

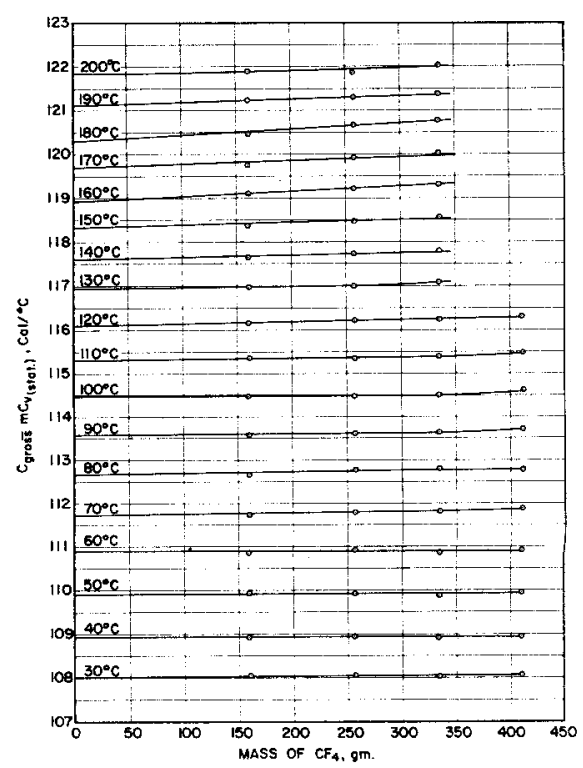

Fig. 1. Colibration of the calorimeter heot capacity by the extrapolation method with tetrafluoromethane. 


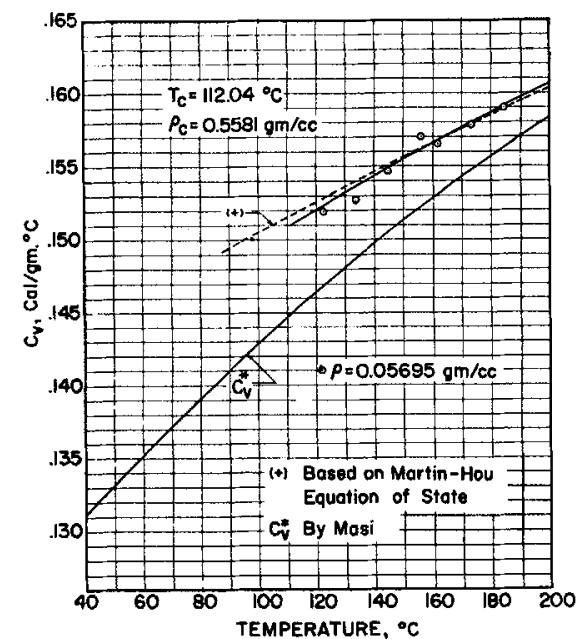

Fig. 2. Constant-volume heat copacity of dichlorodifluoromethane.

capacity estimated from the fundamental frequencies taken from infrared and Raman spectra. It is to be noted that the data points here and later are the result of a smoothing operation that is not shown. For a given density a series of values of $C_{\mathrm{gr}}$ were measured for finite temperature jumps. These values were plotted vs. the average temperature in each jump. A smooth curve was drawn through the points and $\mathrm{Cgr}_{\mathrm{g}}$ read off at even temperatures.

The sample of tetrafluoromethane used was stated to have a purity of at least $99.7 \%$ by volume, as measured by gas chromatography and mass spectrometer analysis.

\section{RESULTS}

Since the heat capacity of the calorimeter is appreciable in comparison with the heat capacity of the gas charged, the accuracy of the gas heat capacity is affected considerably by the calorimeter heat capacity. It is believed that the extrapolation technique determines the heat capacity of the calorimeter to about $0.4 \%$, and that considering all errors the heat capacity of the gas is determined to within $0.8 \%$. This has been verified by first

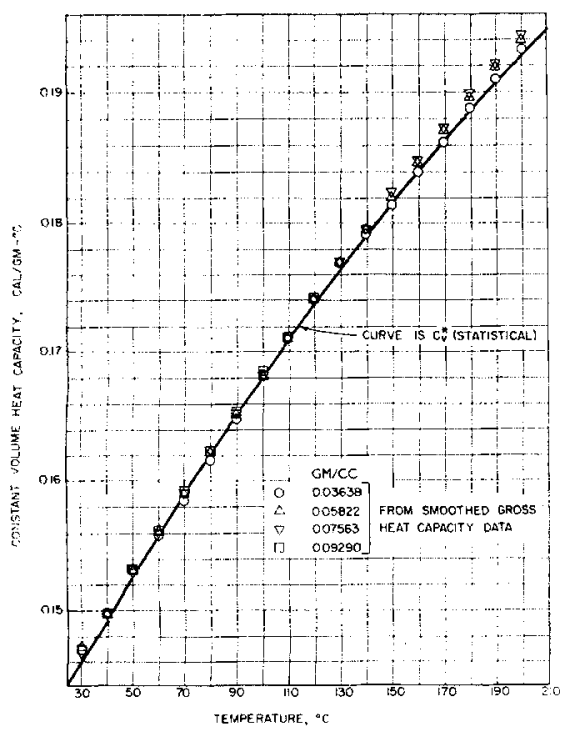

Fig. 3. Heat capacity of gaseous tetrafluoromethone. measuring the heat capacity of dichlorodifluoromethane. This compound has been studied very carefully by Masi (10), so that its $C_{V}^{*}$ is known with a high degree of confidence. The heat capacity of a charge of dichlorodifluoromethane was measured and the data plotted on Figure 2. Since its $C_{V}$ varies appreciably with density, it was necessary to convert the $C_{V}^{*}$ of Masi to $C_{V}$ at the experimental density by the usual relation $d C_{V}=-T V^{2}$ $\left(d^{2} P / d T^{2}\right) d_{\rho}$. Figure 2 shows the $C_{V}$ which results when the conversion is made by an equation of state presented (8) for this compound. The conversion is not considered to be very accurate because it involves the second derivative of the equation of state, but the correction only amounts to $4 \%$ of the answer so the result cannot be too far in error. The agreement of the experimental points with the dashed line $C_{V}$ is good and substantiates the calibration of the calorimeter.

The same data taken for calibration of the calorimeter were used to obtain the constant volume heat capacity of the tetrafluoromethane itself. At each temperature and density the heat capacity of the calorimeter was subtracted from the gross heat capacity to give the heat capacity of the gas on a unit basis by dividing by the mass of gas. The results are shown in the table and in

\section{Table 1. Constant-Volume Heat Capacrty of Tetrafluoromethane}

\begin{tabular}{ccccc} 
& \multicolumn{4}{c}{$C_{V}$, cal./g. ${ }^{\circ} \mathrm{C}}$. \\
$T,{ }^{\circ} \mathrm{C}$. & 0.03638 & 0.05822 & 0.07563 & 0.09290 \\
(Density) & g./cc. & g./cc. & g./cc. & g./cc. \\
& & & & \\
30 & 0.1469 & 0.1469 & 0.1467 & 0.1468 \\
40 & 0.1497 & 0.1497 & 0.1497 & 0.1498 \\
50 & 0.1531 & 0.1530 & 0.1528 & 0.1531 \\
60 & 0.1557 & 0.1561 & 0.1559 & 0.1561 \\
70 & 0.1584 & 0.1588 & 0.1590 & 0.1591 \\
80 & 0.1615 & 0.1622 & 0.1623 & 0.1622 \\
90 & 0.1647 & 0.1650 & 0.1651 & 0.1653 \\
100 & 0.1680 & 0.1680 & 0.1681 & 0.1684 \\
110 & 0.1711 & 0.1709 & 0.1712 & 0.1712 \\
120 & 0.1739 & 0.1740 & 0.1740 & 0.1741 \\
130 & 0.1767 & 0.1767 & 0.1769 & \\
140 & 0.1790 & 0.1793 & 0.1794 & \\
150 & 0.1813 & 0.1819 & 0.1823 & \\
160 & 0.1838 & 0.1845 & 0.1847 & \\
170 & 0.1861 & 0.1870 & 0.1871 & \\
180 & 0.1887 & 0.1895 & 0.1897 & \\
190 & 0.1909 & 0.1918 & 0.1920 & \\
200 & 0.1932 & 0.1939 & 0.1942 &
\end{tabular}

Note: $C_{v}$ data were all calculated from the smoothed gross heat capacity data.

Figure 3. Also on this figure a curve is drawn which represents a statistically calculated $C_{V} *$. Infrared and Raman spectra have been measured by a number of investigators $(2,3,5,6,11,12,13,14)$. After careful study of these data the following set of fundamental vibrational frequencies was chosen; $\nu_{1}=435 \mathrm{~cm}^{-1}$ (degeneracy of 2), $\nu_{2}=630 \mathrm{~cm} .^{-1}$ (degeneracy of 3), $908 \mathrm{~cm}$. (no degeneracy), $y_{4}=1,282 \mathrm{~cm}^{-1}$ (degeneracy of 3 ). The usual rigid-rotator-harmonic-oscillator assumption was made first, and a small correction for anharmonicity and variation of moment of inertia due to stretching was added. The latter was made by the approximation method of Albright (1), and the results are practically identical to his for tetrafluoromethane. The correction amounted to only about $0.5 \%$ of $C_{V}$ itself in the range of the data and less than $1 \%$ even at $700^{\circ} \mathrm{K}$., though a greater uncertainty is introduced at the higher temperatures because 
the correction procedure has not been verified in that range.

Figure 3 shows good agreement between the measured and statistically calculated heat capacity of tetrafluoromethane over the $30^{\circ}$ to $200^{\circ} \mathrm{C}$. range. In view of this agreement the statistical calculations were extended over a much wider range, $140^{\circ}$ to $620^{\circ} \mathrm{K}$, , and the data fitted to an equation

$$
\begin{array}{r}
C_{V}=0.00375+\frac{102.9}{T^{2}}+5.7857 \times 10^{-4} \mathrm{~T}- \\
3.7829 \times 10^{-7} T^{2} \mathrm{cal} . / \mathrm{g} .-{ }^{\circ} \mathrm{K} .
\end{array}
$$

with $T$ in ${ }^{\circ} \mathrm{K}$. This fits the data with a maximum error of only $0.3 \%$. It is worthwhile mentioning that a curve of $C_{V}$ * vs. $T$ undergoes an inflection which cannot be represented precisely with a cubic expansion in positive powers of $T$ as is usually done. It is necessary to use an expansion in reciprocal temperature or some other more complex mathematical function.

\section{ACKNOWLEDGMENT}

The authors are grateful to the E. I. du Pont de Nemours and Company for financial assistance and for supplying the samples of tetrafluoromethane, to the Esso Research and Engineering Company for an equipment grant, and to The University of Michigan Rackham Graduate School for fellowship support.

\section{LITERATURE CITED}

1. Albright, L. F., W. C. Galegar, and K. K. Innes, J. Am. Chem. Soc., 76, 6017 (1954).

2. Bailey, C. R., J. B. Hale, and J. W. Thompson, J. Chem. Phys., 5, 274 ( 1937).

3. Claassen, H. H., ibid., 22, 50 (1954).

4. De Nevers, N. H., and J. J. Martin, A.I.Ch.E. Journal, 6, 43 (1960).

5. Eucken, A., and A. Bertram, Z. Physik. Chem., B31, 361 (1936).

6. Goubeau, J., W. Bues, and F. W. Kampmann, Z. Anorg. Allgem. Chem., 283, 123 (1956).

7. Hwang, Y. T., Ph.D. thesis, Univ. Mich., Ann Arbor, Michigan (Feb., 1961).

8. McHarness, R. C., B. J. Eiseman, Jr., and J. J. Martin, Refrig. Eng, 63, 31 (1955).

9. Martin, J. J., "Progress in International Research on Thermodynamic and Transport Properties," p. 93, Am. Soc. Mech. Engrs., Academic Press, New York (1962).

10. Masi, J. F., J. Am. Chem. Soc., 74, 4738 (1952).

11. Monostori, B., and A. Weber, J. Chem. Phys., 33, 1867 (1960).

12. Plyler, E. K., and W. S. Benedict, J. Res. Natl. Bur. Stds., 47, 202 (1951).

13. Woltz, P. J. H., and A. H. Nielsen, J. Chem. Phys., 20, 307 (1952)

14. Yost, D. M., E. N. Lassertre, and S. T. Gross, ibid., 4, 325 (1936)

Manuscript received March 15, 1963; revision received June 18, 1963; paper accepted June 27, 1963. Paper presented at A.I.Ch.E. New Orleans meeting.

\section{Idealized Theory for Turbulent}

\section{Mixing in Vessels}

RONALD E. ROSENSWEIG

Aveo Research and Advanced Development Division, Wilmington, Massachusetts

The engineer commonly estimates the influence of mixing on process operations by resorting to useful simplifying assumptions. Two such assumptions are the notions of plug flow and well-mixed systems. In the following treatment a means is demonstrated for predicting states of mixing for miscible systems which are intermediate to the above mentioned cases. This analysis utilizes mostly known results of the modern theory of turbulence as based on the statistical description and exploits Kolmogoroff's concept of universal equilibrium (10). The system studied is the continuous flow stirred vessel. Current state of the art regarding such turbulent mixers allows one to predict the power input given knowledge of the mixer geometry, fluid properties, and stirring rate. Power input rates are well correlated for many existing mixers through work such as that of Rushton et al. (12). What has not been adequately known is how to predict the effect of this specified stirring on the mixing job to be done.

The stirred tank mixer characteristically operates up to high Reynolds' numbers based on impeller diameter and tip velocity $\left(\sim 10^{6}\right)$ which should be conducive to approaching universal equilibrium in the Kolmogoroff sense (10). Shinnar (13) has advanced some stimulating conjectures on the nature of emulsion formation in stirred tanks in which he exploits behavior of the velocity spectrum at wave numbers in the dissipative range of eddy sizes. In the present work knowledge of the inertial subrange, a lower wave number region, is exploited.

The dissipation of scalar inhomogeneities is generally pictured to be preceded by cascade down the spectrum. 